\title{
A!
}

This is an electronic reprint of the original article.

This reprint may differ from the original in pagination and typographic detail.

Hold, Christoph; Politis, Archontis; McCormack, Leo; Pulkki, Ville

\section{Spatial Filter Bank Design in the Spherical Harmonic Domain}

Published in:

29th European Signal Processing Conference, EUSIPCO 2021

DOI:

10.23919/EUSIPCO54536.2021.9616091

Published: 01/12/2021

Document Version

Peer reviewed version

Please cite the original version:

Hold, C., Politis, A., McCormack, L., \& Pulkki, V. (2021). Spatial Filter Bank Design in the Spherical Harmonic Domain. In 29th European Signal Processing Conference, EUSIPCO 2021 (pp. 106 - 110). (European Signal Processing Conference). IEEE. https://doi.org/10.23919/EUSIPCO54536.2021.9616091

This material is protected by copyright and other intellectual property rights, and duplication or sale of all or part of any of the repository collections is not permitted, except that material may be duplicated by you for your research use or educational purposes in electronic or print form. You must obtain permission for any other use. Electronic or print copies may not be offered, whether for sale or otherwise to anyone who is not an authorised user. 


\title{
Spatial Filter Bank Design in the Spherical Harmonic Domain
}

\author{
Christoph Hold $^{1 \star}$, Archontis Politis ${ }^{2}$, Leo McCormack ${ }^{1}$, Ville Pulkki ${ }^{1}$ \\ ${ }^{1}$ Department of Signal Processing and Acoustics, Aalto University, Espoo, Finland \\ ${ }^{2}$ Department of Information Technology and Communication Sciences, Tampere University, Finland \\ ${ }^{\star}$ Christoph.Hold@aalto.fi
}

\begin{abstract}
A fairly recent development in spatial audio is the concept of dividing a spherical sound field into several directionally-constrained regions, or sectors. Therefore, the sphere is spatially partitioned into components that should ideally reconstruct the unit sphere. When distributing such sectors uniformly on the sphere, their set makes up a bank of spatial filters, i. e. a spatial filter bank. These sectors can be conveniently designed in the spherical harmonic domain such that each sector preserves the local properties of the acoustic energy-density. These traits have enabled recent improvements in the parameterization of higher-order Ambisonics, e.g. for spatial audio reproduction, multi-source analysis, and sound field visualization. However, when using a set of these sectors as a spatial filter bank, their spatial interaction incurs a scaling error if the reconstructed sound field is not properly compensated. This paper presents the methodology for designing a set of spatial filters in the spherical harmonic domain, which uniformly partition the sphere. Furthermore, a new corresponding compensation factor is derived enabling amplitude or energy preservation of the input sound field. This allows the implementation of a novel spatial filter bank in the spherical harmonic domain.
\end{abstract}

Index Terms-Spherical Harmonics, Ambisonics, Spatial Audio, Spatial Filter Bank

\section{Motivation}

Filters permit the ability to constrain a domain, such that only a certain part of it is observable. With a collection of filters one may therefore subdivide a domain into several parts. In the time-frequency domain, e.g. , a set of time-frequency filters can make up a filter bank that allows analysis of separate parts of the time-frequency spectral domain [1], [2].

Generalizing this concept and applying it to the spatial domain along a sphere, i.e. the angular domain, a set of spatial filters may divide the sphere into its partitions. This could lead directly to the partitioning of the surface area, e.g. by tessellation [3]. Spatial filtering of sound fields is also termed beamforming and a set of beamformers as a spatial filter bank was explored for speech enhancement in [4]. Related is the technique of Spatial PCM Sampling [5], which describes a spatial audio recording as a set of discrete (virtual) microphone outputs. However, spherical functions can also be described by their angular spectrum in the spherical harmonic $(\mathrm{SH})$ domain and thus spatial filtering may be carried out directly in the $\mathrm{SH}$ domain [6]. This applies directly to spatial sound fields and

This project has received funding from the Fraunhofer IIS and from the Academy of Finland. a spatial beamformer for spherical microphone arrays is also elegantly designed in the spherical harmonics domain [7], [8].

Regarding sound fields, an emerging methodology in spatial audio is the concept of dividing the sphere into multiple partitions directly in the spherical harmonic (or Ambisonics) domain. These spherical sectors can be designed such that they spatially constrain the sound field to local estimates of the acoustic energy-density, thus allowing to form sets of spatiallyconstrained basis functions [9]. Therefore, those sectors enable estimates of the sound field intensity contribution per region [9], e. g. for directional spatial analysis of reverberation [10]-[12]. This principle furthermore constitutes one way of improving methods based upon models that rely on estimating sound field (pseudo-)intensity, e.g. for direction of arrival (DOA) estimation [13], [14]. Without further assumptions, those models typically extract solely a single intensity vector and are therefore only able to describe a single point source accurately. Subdividing the sound field into sectors thus allows for multiple DOA estimates, potentially increasing resolution and minimizing uncertainty [15].

This subdivision of the input sound field into sectors has represented a convenient way to extend traditionally first-order-only parametric sound field reproduction methods, such as spatial impulse response rendering (SIRR) [16] and directional audio coding (DirAC) [17], utilizing the higher spatial resolution afforded by higher-order $\mathrm{SH}$ input. Thereby extracting multiple spatially localized active intensity vectors leads directly to the sector-based spatial audio processing explored in [18], [19]. It is therefore crucial that merging the individual sectors results in reconstructing the original spherical sound field properties.

Typically, filter banks may suffer from a non-uniform amplitude or energy total response as well as scaling issues. Spatially uniform reconstruction is generally provided by sufficient uniform covering [18]. However, due to the limited spatial bandwidth of designs in the spherical harmonics domain, the sector designs employed in the aforementioned works will inevitably result in a scaled version of the unit sphere; often varying drastically depending on the chosen sector design patterns. Therefore, there is a systematic scaling error between the input and output sound fields; and since certain applications may benefit from arbitrary sector patterns, e. g. higher-order cardioids, they need a flexible and efficient compensation technique. 


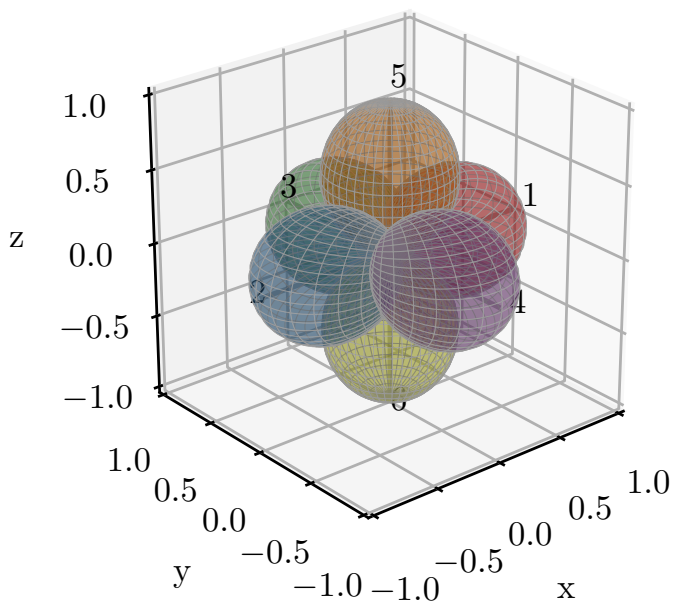

Figure 1. Sector layout consisting of uniformly distributed normalized $\max \mathbf{r}_{E}$ sector beamformers, capable of preserving amplitude up to $N=2$.

While the literature has previously reported briefly on the topic of compensation factors for energy preservation [18, Sec. Appendix], it has not yet been investigated for amplitude preservation. Other previous works that did not include an analytic solution, but rather a least-squares approximation, will also vary in their performance when employing arbitrary sector patterns [15]. Therefore, this paper demonstrates a design methodology dividing the sphere with a set of beamformers, forming a spatial filter bank in the SH domain. An analytical formulation is derived that restores correct scaling dependent on the beamformer design.

\section{A. Objectives}

There are two main objectives for the reconstruction of a unit sphere divided by spatial weighting. First, reconstruction of the amplitude, or reconstruction of the energy (domain of squares).

This means, for all points on the unit sphere $\Omega=[\phi, \theta]$, with azimuth $\phi$ and zenith/colatitude $\theta$, the sum over the weighting $w$ of sectors $\xi=1, \ldots, J$ shall return the unit sphere.

The objective translates to preserving amplitude

$$
\sum_{\xi=1}^{J} \beta_{\mathrm{A}} w_{\xi}(\Omega)=1, \quad \forall \Omega \in \mathbb{S}^{2},
$$

or preserving energy

$$
\sum_{\xi=1}^{J} \beta_{\mathrm{E}} w_{\xi}^{2}(\Omega)=\sum_{\xi=1}^{J}\left[\sqrt{\beta_{\mathrm{E}}} w_{\xi}(\Omega)\right]^{2}=1, \quad \forall \Omega \in \mathbb{S}^{2},
$$

respectively. Choosing a uniform sector layout simplifies the reconstruction factors $\beta$ to a single sector independent scalar value. As introduced before, a spatial weighting $w$ will be chosen dependent on the application and conveniently as beamformers in the spherical harmonic domain, which requires reconstruction scaling correction. The factors $\beta$ are the main focus of this document, as they restore scaling to unity.

\section{BACKGROUND}

This paper uses the orthonormal spherical harmonic basis functions $Y_{n}^{m}$ of order $n$ and degree $m$ given as [20, Eq. (6.20)]

$$
Y_{n}^{m}(\Omega)=\sqrt{\frac{2 n+1}{4 \pi} \frac{(n-m) !}{(n+m) !}} P_{n}^{m}(\cos (\theta)) e^{i m \phi},
$$

where $P_{n}^{m}$ is the associated Legendre polynomial including the Condon-Shortley phase. The corresponding spherical harmonic transform and inverse transform are given in [21, Eq. (1.40) and (1.41)], where $N$ is the maximum SH order.

Parseval's relation of the functions $f, g$ relates the discrete and spherical harmonic domain as [21, Eq. (1.44)]

$$
\int_{\mathbb{S}^{2}} f(\Omega) g^{*}(\Omega) d \Omega=\sum_{n=0}^{N} \sum_{m=-n}^{n} f_{n m} g_{n m}^{*}=\mathbf{g}_{n m}^{\mathrm{H}} \mathbf{f}_{n m} .
$$

The spherical harmonics addition theorem is given as [21, Eq. (1.26)]

$$
\sum_{m=-n}^{n}\left[Y_{n}^{m}(\Omega)\right]^{*} Y_{n}^{m}\left(\Omega^{\prime}\right)=\frac{2 n+1}{4 \pi} P_{n}(\cos (\Theta))
$$

where the angle $\Theta=\angle\left(\Omega, \Omega^{\prime}\right)$ and $P_{n}$ the degree $n$ Legendre polynomial.

Due to orthonormality, the integration of spherical harmonics

$$
\int_{\mathbb{S}^{2}} Y_{n}^{m}(\Omega) d \Omega=\sqrt{4 \pi} \delta_{n 0}
$$

vanishes for all components, except $n=m=0$ [21, Eq. (1.22)].

The latter can be carried out as a numerical integration by quadrature grids, e. g. with uniform nodes at $\Omega_{j}$ on a spherical t-design [22], [23] as

$$
\int_{\mathbb{S}^{2}} f(\Omega) d \Omega=\frac{4 \pi}{J} \sum_{j=1}^{J} f\left(\Omega_{j}\right) .
$$

Equation (6) and Eq. (7) give

$$
\begin{gathered}
\int_{\mathbb{S}^{2}} Y_{n}^{m}(\Omega) d \Omega=\frac{4 \pi}{J} \sum_{j=1}^{J} Y_{n}^{m}\left(\Omega_{j}\right) \\
\sum_{j=1}^{J} Y_{n}^{m}\left(\Omega_{j}\right)=\frac{J}{\sqrt{4 \pi}} \delta_{n 0} .
\end{gathered}
$$

For the spatial weighting, axisymmetric spherical array beam patterns can be described by their $N$ beamforming weights or modal weighting coefficients $c_{n}$ as [21, Eq. (5.24)]

$$
w(\Theta)=\sum_{n=0}^{N} \frac{2 n+1}{4 \pi} c_{n} P_{n}(\cos (\Theta)) .
$$




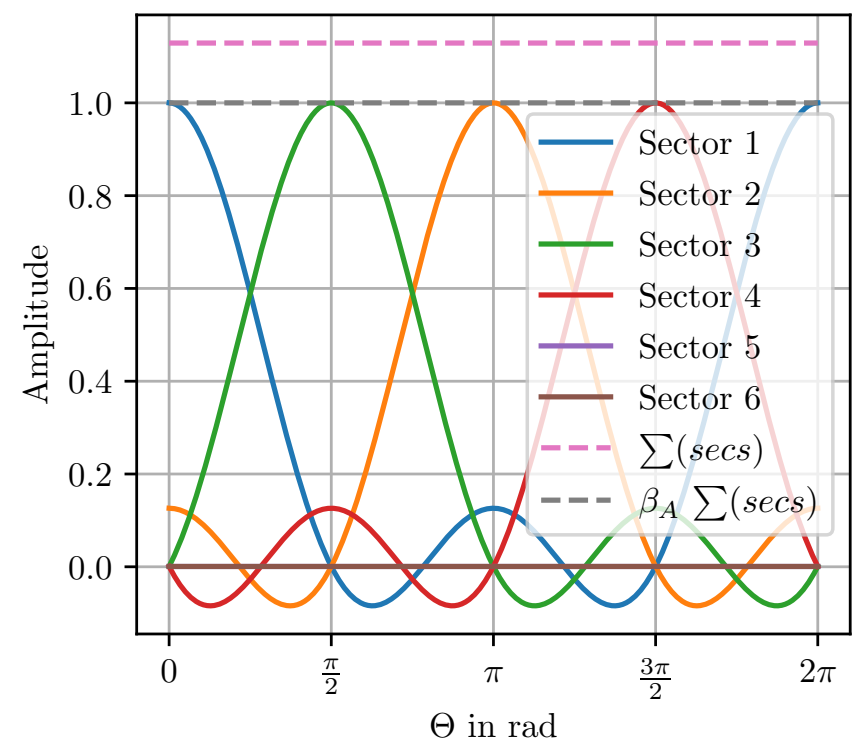

Figure 2. Cross-section $(\theta=\pi / 2$, i. e. $x$ - $y$-plane $)$ of a spatial filterbank with second order $\max \mathbf{r}_{E}$ sector beamformers and their amplitude summation, with and without the compensation factor $\beta_{\mathrm{A}}$.

\section{METHODS}

\section{A. Number of Sectors}

The minimal number of sectors $J$ necessary for reconstruction depends on the numerical integration quadrature in Eq. (7). Energy preservation demands for a quadrature capable of integrating $f(\Omega)$ of degree $2 N$. Amplitude preservation only requires a quadrature for degree $N+1$. However, a quadrature of higher order also integrates correctly, meaning for every order $N>0,2 N>=N+1$, hence, a quadrature allowing energy preservation also allows amplitude preservation. This study uses uniformly distributed sector steering directions $\Omega_{\xi}=\Omega_{j}$ further on.

\section{B. Spatial Weighting of Selected Patterns}

An axisymmetric beamformer is characterized by its order dependent weighting $c_{n}$, compare Eq. (9). The simplest beamformer of that kind is the normalized plane-wave decomposition (PWD), also called max DI, which results in a higher order hyper-cardioid pattern. The modal weighting is a constant factor of [21, Eq. (6.10).]

$$
c_{n}=\frac{4 \pi}{(N+1)^{2}} .
$$

A higher order cardioid pattern might be expressed in the discrete domain as

$$
w(\Theta)=\left(A+(1-A) \cos (\Theta)^{N} \text {, with } A=0.5 .\right.
$$

This leads to the axisymmetric beamformer in the spherical harmonics domain with

$$
c_{n}=\frac{4 \pi N ! N !}{(N+n+1) !(N-n) !} .
$$

A popular choice in spatial audio is the $\max \mathbf{r}_{E}$ pattern, with similar properties as super-cardioids, however, the modal weighting is directly given as [24, Eq. (10)]

$$
c_{n}=P_{n}\left(\cos \left(\frac{2.4068}{N+1.51}\right)\right)
$$

where the two constants were numerically optimized. The criterion, however, did not ensure unit amplitude in steering direction. While strictly speaking not necessary for the derivation found in this document, any axisymmetric pattern may be normalized in steering direction by a weighted band-limited spatial Dirac $a_{n o r m}=\sum_{n=0}^{N} c_{n} \frac{2 n+1}{4 \pi}$. Such sector pattern for $N=2$ is shown in Fig. 1 and 2 and used exemplary throughout this document.

\section{Amplitude Preservation Factor}

Spatial weighting summed over all sectors $\xi$ simplifies because of uniform pattern distribution to

$$
\sum_{\xi=1}^{J} w_{\xi}(\Omega)=\sum_{\xi=1}^{J} w\left(\alpha_{\xi}\right)
$$

where $\alpha_{\xi}=\angle\left(\Omega_{\xi}, \Omega\right)$. With Eq. (9) this becomes

$$
=\sum_{\xi=1}^{J} \sum_{n=0}^{N} c_{n} P_{n}\left(\cos \left(\alpha_{\xi}\right)\right)
$$

Comparing with Eq. (5) and reordering gives

$$
\begin{aligned}
\sum_{\xi=1}^{J} \sum_{n=0}^{N} c_{n} \sum_{m=-n}^{n}\left[Y_{n}^{m}(\Omega)\right]^{*} Y_{n}^{m}\left(\Omega_{\xi}\right)= \\
\sum_{n=0}^{N} c_{n} \sum_{m=-n}^{n}\left[Y_{n}^{m}(\Omega)\right]^{*} \sum_{\xi=1}^{J} Y_{n}^{m}\left(\Omega_{\xi}\right)
\end{aligned}
$$

Evaluating the sum over $J$ as in Eq. (8), due to orthogonality of spherical harmonics, components $n>0$ vanish and only the zeroth order components remain

$$
\sum_{\xi=1}^{J} w_{\xi}(\Omega)=c_{0} Y_{0}^{0}(\Omega)^{*} \frac{J}{\sqrt{4 \pi}}
$$

As $Y_{0}^{0}(\Omega)=\sqrt{4 \pi}^{-1}$, the sum over the amplitude of all sectors becomes

$$
\sum_{\xi=1}^{J} w_{\xi}(\Omega)=c_{0} \frac{J}{4 \pi}
$$

Under the objective Eq. (1) this leads directly to the compensation factor for amplitude preservation

$$
\beta_{\mathrm{A}}=\frac{4 \pi}{c_{0} J}
$$




\section{Energy Preservation Factor}

This is shown in [18, Appendix], however, there with a different definition of Eq. (9), and hence not with the beamforming modal weights $c_{n}$.

Analogues to the amplitude factor, but substituting the squared pattern $w^{2}=d$, the sum over all sectors results in

$$
\sum_{\xi=1}^{J} w^{2}\left(\alpha_{\xi}\right)=\sum_{\xi=1}^{J} d\left(\alpha_{\xi}\right)=d_{0} \frac{J}{4 \pi} .
$$

Under the objective Eq. (2), the compensation factor for energy preservation may be formulated as

$$
\beta_{\mathrm{E}}=\frac{4 \pi}{d_{0} J}
$$

\section{E. Recovering From SH Beampattern}

In case the modal weighting $c_{n}$ is unavailable, the compensation factors can be alternatively retrieved from any axisymmetric SH sector beampattern vector $\mathbf{w}_{n m}$ in sector steering direction $\Omega_{\xi}$

$$
\mathbf{w}_{n m}=\operatorname{diag}_{N}\left(\mathbf{c}_{n}\right) \mathbf{y}_{n}^{m}\left(\Omega_{\xi}\right)^{\mathrm{H}} .
$$

The operator $\operatorname{diag}_{N}(\cdot)$ expands every vector (order) entry $m$ times to a diagonal matrix, therefore

$$
\beta_{\mathrm{A}}=\left[\sum_{\xi=1}^{J} w_{\xi}(\Omega)\right]^{-1}=\frac{4 \pi}{c_{0} J}=\frac{\sqrt{4 \pi}}{w_{00} J} .
$$

Re-substituting $d=w^{2}$ and using Parseval's relation Eq. (4), the modal weighting is expanded to

$$
d_{0}=Y_{0}^{0}(\Omega)^{-1} \int_{\mathbb{S}^{2}} w^{2}(\Omega) Y_{0}^{0}(\Omega) d \Omega=\mathbf{w}_{n m}^{\mathrm{H}} \mathbf{w}_{n m},
$$

yielding the alternative form

$$
\beta_{\mathrm{E}}=\left[\sum_{\xi=1}^{J} w_{\xi}^{2}(\Omega)\right]^{-1}=\frac{4 \pi}{d_{0} J}=\frac{4 \pi}{\mathbf{w}_{n m}^{\mathrm{H}} \mathbf{w}_{n m} J}=\frac{Q_{c}}{J},
$$

with the directivity factor

$$
Q_{c}=\frac{4 \pi}{\int_{\mathbb{S}^{2}} w^{2}(\Omega) d \Omega}=\frac{4 \pi}{\mathbf{w}_{n m}^{\mathrm{H}} \mathbf{w}_{n m}} .
$$

An amplitude independent directivity factor can be found in [21, Eq. (5.30)].

Another interesting observation follows directly from Eq. (23) and Eq. (25), in combination with the orthogonality property of spherical harmonics. As a max DI beamformer (normalized PWD) is achieved by constant modal weighting $c_{n}=\frac{4 \pi}{(N+1)^{2}}$, the factors $\beta_{\mathrm{A}}$ and $\beta_{\mathrm{E}}$ become equivalent

$$
\beta_{\mathrm{A}, \max \mathrm{DI}}=\frac{4 \pi}{c_{0} J}=\frac{4 \pi}{4 \pi /(N+1)^{2} J}=\frac{(N+1)^{2}}{J},
$$

and

$$
\begin{aligned}
& \beta_{\mathrm{E}, \max \mathrm{DI}}=\frac{4 \pi}{\mathbf{w}_{n m}^{\mathrm{H}} \mathbf{w}_{n m} J}= \\
& \frac{4 \pi}{\left(4 \pi /(N+1)^{2}\right)^{2}(N+1)^{2} /(4 \pi) J}=\frac{(N+1)^{2}}{J} .
\end{aligned}
$$

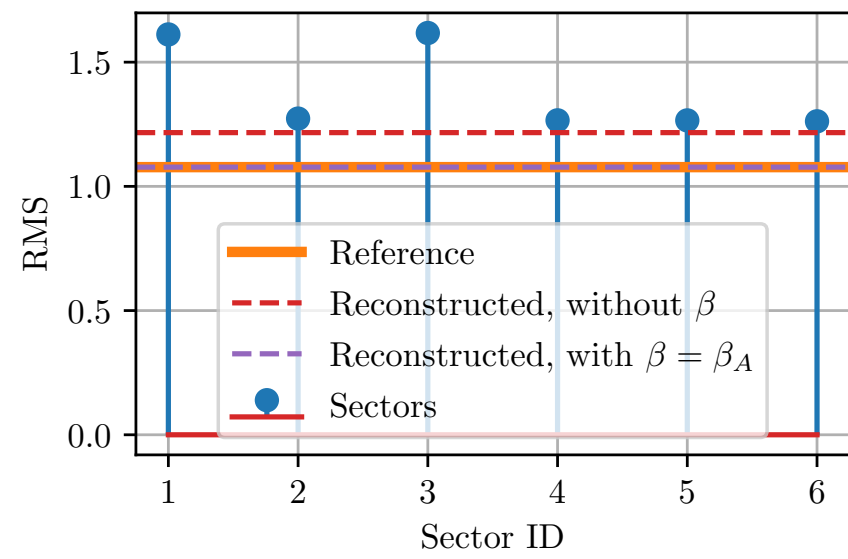

Figure 3. RMS estimates for $\max \mathbf{r}_{E}$ sectors of second order. Shown is a diffuse unit sound field plus two unit plane-waves overlaying in first and third sector. Shown is amplitude reconstruction, with and without compensation $\beta=\beta_{\mathrm{A}}$, compared to a reference.

\section{Evaluation AND Discussion}

Ambisonic signals divided into sectors through a spatial filter bank need a compensation factor in order to mitigate a reconstruction scaling error, which is dependent on the chosen design. Evaluating amplitude reconstruction of second order $\operatorname{maxr}_{E}$ patterns according to Eq. (1), Fig. 2 shows that the individual sectors depicted in Fig. 1 sum up correctly to unit amplitude when considering the compensation factor $\beta_{\mathrm{A}}$. The amplitude reconstruction error as well as its mitigation by applying $\beta_{\mathrm{A}}$ is shown for varying order in table I.

Table I

AMPLITUdE RECONSTRUCTION RMS ERROR IN DB FOR $\max \mathbf{r}_{E}$ PATTERNS

\begin{tabular}{c|cc} 
SH Order & $\beta=1$ & $\beta=\beta_{\mathrm{A}}$ \\
\hline$N=1$ & 3.339 & $<0.000$ \\
$N=2$ & 1.053 & $<0.000$ \\
$N=3$ & 2.724 & $<0.000$ \\
$N=4$ & -0.754 & $<0.000$ \\
$N=5$ & 2.370 & $<0.000$ \\
$N=6$ & -0.112 & $<0.000$ \\
$N=7$ & 1.238 & $<0.000$ \\
$N=8$ & 1.807 & $<0.000$ \\
$N=9$ & 2.008 & $<0.000$ \\
$N=10$ & 1.768 & $<0.000$
\end{tabular}

A numerical evaluation of $\beta_{\mathrm{A}}$ (Eq. (23)) comprises inverting the sum of sectors ad-hoc in the discrete domain on a sufficiently dense sampling grid, i.e. numerically solving Eq. (23) for $\beta_{\mathrm{A}}$. Therefore, we stack the SH sector patterns $w_{n m, \xi}$, evaluated at the grid points $\left[q_{1}, \ldots, Q\right]$ by the inverse spherical harmonic transform into a matrix $\mathbf{S} \in \Re^{Q \times J}$. The least-squares solution for sector weighting resulting in unit amplitude in each sampling point (written as $[1, \ldots, 1]^{T}$ ) is then given as weights per sector $\beta_{\mathrm{A}, \xi}$ stacked to a vector $\mathbf{b}$

$$
\mathbf{b}=\mathbf{S}^{\dagger}[1, \ldots, 1]^{T}
$$


where $(\cdot)^{\dagger}$ denotes the Moore-Penrose pseudo-inverse. In case of a uniform layout the entries of $\mathbf{b}$ are equal and correspond to the scalar factor $\beta_{\mathrm{A}}$. The unit sphere reconstruction was tested and confirmed within numerical accuracy for all sector patterns shown in this work and for orders $N=1, \ldots, 10$. Table I displays the amplitude scaling error for a maxr $\mathbf{r}_{E}$ sector pattern, varying along $\mathrm{SH}$ orders. It highlights the importance for compensation and confirms the accuracy of $\beta_{\mathrm{A}}$ for amplitude recovery. We furthermore numerically confirmed the previously derived energy preservation factor $\beta_{\mathrm{E}}$ as in Eq. (25). A reference implementation can be found in [25].

In signal space, Fig. 3 shows the results of an $\mathrm{SH}$ input signal $\sigma_{n m \text {,in }}(N=2)$ comprised of a unit amplitude diffuse sound field plus two unit amplitude plane-waves impinging onto the first and third sector. All signals originated from independent $3 \mathrm{~s}\left(f_{s}=48 \mathrm{kHz}\right)$ realizations of noise with standard normal distribution. Therefore, the root-mean-squared (RMS) value in each sector $s\left(\Omega_{j}\right)=\mathbf{w}_{n m, j}^{\mathrm{H}} \boldsymbol{\sigma}_{n m \text {,in }}$ should be close to one, except in sectors with additional plane-wave contributions, where the RMS should fall close to $\sqrt{2}$. Deviations are expected not only because of randomness but also because of the limited $\mathrm{SH}$ order, which causes the sector signals to interfere. We will assume the RMS directly obtained from the zeroth order of the input signal as the reference for reconstruction. The reconstructed RMS from the sector signals according to Eq. (1) is then compared to this reference.

Fig. 3 reports that each sector estimates its expected contribution. It also shows that neglecting the reconstruction scaling (i. e. $\beta=1$ ) results in a significant error. Incorporating the factor $\beta_{\mathrm{A}}$ for amplitude preservation results in numerically accurate RMS reconstruction, for all sector patterns. This highlights again the usefulness of the presented spatial filterbank, as it allows to not only estimate spatially constrained RMS measures, but also recover the total RMS from its spatial parts.

\section{CONCLUSION}

This paper showed a methodology to partition a spherical sound field into spatially constrained sectors of varying shape. It therefore demonstrated the design of spatial filter banks in the spherical harmonics domain which can be applied directly to Ambisonic signals. We furthermore highlighted the reconstruction error of such filter bank designs and derived corresponding compensation factors for restoring amplitude and energy preservation. The newly derived compensation factor was confirmed to preserve the sound fields amplitude obtained from beamformer outputs. The results form a basis for fostering future work, e.g. in parametric spatial audio.

\section{REFERENCES}

[1] M. Vetterli, "A Theory of Multirate Filter Banks," IEEE Transactions on Acoustics, Speech, and Signal Processing, vol. 35, no. 3, pp. 356-372, 1987.

[2] P. P. Vaidyanathan, "Multirate Digital Filters, Filter Banks, Polyphase Networks, and Applications: A Tutorial," Proceedings of the IEEE, vol. 78, no. 1, pp. 56-93, 1990.

[3] B. Beckers and P. Beckers, "A general rule for disk and hemisphere partition into equal-area cells," Computational Geometry: Theory and Applications, vol. 45, no. 7, pp. 275-283, 2012.
[4] Z. Yermeche, P. Cornelius, N. Grbić, and I. Claesson, "Spatial filter bank design for speech enhancement beamforming applications," 2004 Sensor Array and Multichannel Signal Processing Workshop, no. I, pp. 557-560, 2004.

[5] A. Farina, A. Amendola, L. Chiesi, A. Capra, and S. Campanini, "Spatial PCM sampling: A new method for sound recording and playback," in Proceedings of the AES International Conference, 2013, pp. 272-283.

[6] K. Zhou, H. Bao, and J. Shi, "3D surface filtering using spherical harmonics," CAD Computer Aided Design, vol. 36, no. 4, pp. 363-375, 2004.

[7] J. Atkins, "Robust beamforming and steering of arbitrary beam patterns using spherical arrays," IEEE Workshop on Applications of Signal Processing to Audio and Acoustics, pp. 237-240, 2011.

[8] B. Rafaely, "Spatial sampling and beamforming for spherical microphone arrays," in 2008 Hands-free Speech Communication and Microphone Arrays, Proceedings, HSCMA 2008, 2008, pp. 5-8.

[9] A. Politis and V. Pulkki, "Acoustic intensity, energy-density and diffuseness estimation in a directionally-constrained region," arXiv, no. 609.03409, 2016, http://arxiv.org/abs/1609.03409. [Online]. Available: http://arxiv.org/abs/1609.03409

[10] M. Berzborn, M. Nolan, E. Fernandez-Grande, and M. Vorländer, "On the directional properties of energy decay curves," in Proceedings of the International Congress on Acoustics, vol. 2019-September, no. 1, 2019, pp. 4043-4050.

[11] B. Alary, P. Massé, V. Välimäki, and M. Noisternig, "Assessing the Anisotropic Features of Spatial Impulse Responses," Proceedings of the EAA Spatial Audio Signal Processing Symposium, pp. 43-48, 2019.

[12] P. Massé, T. Carpentier, O. Warusfel, and M. Noisternig, "Denoising directional room impulse responses with spatially anisotropic late reverberation tails," Applied Sciences (Switzerland), vol. 10, no. 3, 2020.

[13] D. P. Jarrett, E. A. Habets, and P. A. Naylor, "3D Source localization in the spherical harmonic domain using a pseudointensity vector," in European Signal Processing Conference, 2010.

[14] A. H. Moore, C. Evers, and P. A. Naylor, "Direction of Arrival Estimation in the Spherical Harmonic Domain Using Subspace Pseudointensity Vectors," IEEE/ACM Transactions on Audio, Speech, and Language Processing, vol. 25, no. 10, pp. 1956-1968, oct 2017.

[15] L. McCormack, S. Delikaris-Manias, A. Politis, D. Pavlidi, A. Farina D. Pinardi, and V. Pulkki, "Applications of spatially localized activeintensity vectors for sound-field visualization," AES: Journal of the Audio Engineering Society, vol. 67, no. 11, pp. 840-854, 2019.

[16] J. Merimaa and V. Pulkki, "Spatial impulse response rendering I: Analysis and synthesis," AES: Journal of the Audio Engineering Society, vol. 53 , no. 12, pp. 1115-1127, 2005

[17] V. Pulkki, "Spatial Sound Reproduction with Directional Audio Coding," Journal of Audio Engineering Society, vol. 55, no. 6, pp. 503-516, 2007.

[18] A. Politis, J. Vilkamo, and V. Pulkki, "Sector-Based Parametric Sound Field Reproduction in the Spherical Harmonic Domain," IEEE Journal on Selected Topics in Signal Processing, vol. 9, no. 5, pp. 852-866, 2015.

[19] L. McCormack, V. Pulkki, A. Politis, O. Scheuregger, and M. Marschall, "Higher-Order Spatial Impulse Response Rendering: Investigating the Perceived Effects of Spherical Order, Dedicated Diffuse Rendering, and Frequency Resolution," Journal of the Audio Engineering Society, vol. 68 , no. 5, pp. 338-354, jun 2020.

[20] E. G. Williams, Fourier Acoustics. Academic Press, 1999

[21] B. Rafaely, Fundamentals of Spherical Array Processing, ser. Springer Topics in Signal Processing. Cham: Springer International Publishing, 2019 , vol. 16

[22] R. H. Hardin and N. J. Sloane, "McLaren's improved snub cube and other new spherical designs in three dimensions," Discrete and Computational Geometry, vol. 15, no. 4, pp. 429-441, 1996.

[23] M. Gräf and D. Potts, "On the computation of spherical designs by a new optimization approach based on fast spherical Fourier transforms," Numerische Mathematik, vol. 119, no. 4, pp. 699-724, 2011.

[24] F. Zotter and M. Frank, "All-Round Ambisonic Panning and Decoding," Journal of the Audio Engineering Society, vol. 60, 2012.

[25] C. Hold, Amplitude and Energy Preserving Sector Design Reference Implementation, 2021 (accessed Feb 20), https://github.com/chris-hld/ spaudiopy/blob/sector_design/examples/Sector_design.ipynb. [Online]. Available: https://github.com/chris-hld/spaudiopy/blob/sector_design/ examples/Sector_design.ipynb 